\title{
Endogamia e Limite de Seleção em Populações Selecionadas Obtidas por Simulação
}

\author{
Fernanda Cristina Breda ${ }^{2}, 6$, Ricardo Frederico Euclydes ${ }^{3}$, Carmen Silva Pereira ${ }^{4}$, \\ Robledo de Almeida Torres ${ }^{3,6}$, Paulo Luiz Souza Carneiro ${ }^{5}$, José Lindenberg Rocha \\ Sarmento ${ }^{2,6}$, Rodolpho de Almeida Torres Filho ${ }^{2,6}$, Antonia Kécya França Moita ${ }^{2,6}$
}

\begin{abstract}
RESUMO - Objetivou-se, com este trabalho, avaliar o comportamento do coeficiente de endogamia e do limite de seleção considerando população-base selecionada. Utilizou-se o programa GENESYS para a simulação do genoma constituído de uma única característica quantitativa com valor de herdabilidade igual a 0,40, população-base, população inicial e populações sob seleção. Foram consideradas três gerações distintas como gerações bases, gerações zero $\left(G_{0} P B\right)$, três $\left(G_{3} P B\right)$ e sete $\left(G_{7} P B\right)$. As populações foram selecionadas a partir dos valores genéticos obtidos pelo melhor preditor linear não-viesado (BLUP) e com base no desempenho individual, sendo considerados: a) dois tamanhos efetivos de população $\left(\mathrm{N}_{\mathrm{e} 1}=38,09\right.$ e $\left.\mathrm{N}_{\mathrm{e} 2}=88,88\right)$ e b) dois sistemas de acasalamentos dos reprodutores selecionados (reprodutores acasalados aleatoriamente - RAA e exclusão de irmãos completos - EIC). Os parâmetros avaliados foram: coeficiente médio de endogamia, percentagem de locos fixados favoravelmente e limite de seleção. A não-utilização da população-base verdadeira $\left(\mathrm{G}_{0} \mathrm{~PB}\right)$ subestimou os coeficientes de endogamia. As populações que utilizaram as gerações três e sete como base apresentaram as mesmas taxas de fixação de alelos favoráveis e os mesmos valores do limite de seleção das populações que consideraram a $\mathrm{G}_{0} \mathrm{~PB}$.
\end{abstract}

Palavras-chave: endogamia, população base, seleção, tamanho efetivo da população

\section{Inbreeding and Selection Limit in Selected Population Obtained by Simulation}

\begin{abstract}
Objective was to evaluate the behavior average inbreeding coefficient associated to the selection limit considering selected population as base population. GENESYS program was used for simulation of the genome (one trait of $h^{2}=0.40$ ), base population, initial population and populations under selection. Three different generations were considered as base generations, zero $\left(G_{0} P B\right)$, three $\left(G_{3} P B\right)$ and seven $\left(G_{7} P B\right)$. Populations were selected based on best linear unbiased predictor (BLUP) and on individual phenotype in which were considered: a) two effective population sizes $\left(\mathrm{N}_{\mathrm{e} 1}=38.09\right.$ and $\mathrm{N}_{\mathrm{e} 2}=88.88$ ) and b) two mating systems (random mating - RAA and exclusion of full sibs - EIC). Evaluated parameters were: average inbreeding coefficient, fixation of favorable alleles and selection limit. Selected populations considered as true base population resulted in underestimated inbreeding coefficient. Populations that used generations three and seven as true base population presented the same rates of fixation of favorable alleles and the same values for selection limit as those of populations that considered $G_{0} P B$.
\end{abstract}

Key Words: inbreeding, base population, selection, population effective sizes

\section{Introdução}

A endogamia é um sistema de acasalamento em que os indivíduos mais aparentados entre si que a média da população são utilizados como pais da próxima geração. Tem como principal efeito genético o aumento da homozigose e o aparecimento de genes recessivos que, geralmente, provocam alguma alteração na média do mérito individual.

A medida do aumento da homozigose é dada pelo coeficiente de endogamia, que se torna expressivo quando há especificação de algum ponto no passado, em que os ancestrais não são mais procurados, de modo que todos os genes presentes na população passam a ser não-idênticos por descendência. Esse ponto representa a população-base e, por definição, tem coeficiente de endogamia igual a zero (Falconer, 1987).

O coeficiente de endogamia depende do tamanho efetivo da população e, quanto menor for o tamanho da população, em gerações anteriores, maior será o número de ancestrais comuns e maior será o coeficiente de endogamia.

Faria (2002), em estudo sobre a estrutura genética das populações zebuínas brasileiras, observou grande

\footnotetext{
${ }^{1}$ Parte da tese de mestrado apresentada à UFV, para obtenção do título de mestre. Apoio CNPq.

${ }^{2}$ Estudantes de Pós-graduação. E. mail: ferbreda@hotmail.com

3 Professor do DZO/UFV, Viçosa - MG, CEP: 36571-000.

4 Professor do Departamento de Zootecnia da Escola de Veterinária da Universidade Federal de Minas Gerais.

${ }^{5}$ Professor do Departamento de Ciências Biológicas da Estadual do Sudoeste da Bahia.

${ }^{6}$ Bolsista do CNPq.
} 
aumento da variância do número de progênies por reprodutor no período de 1994 a 1998, refletindo o uso intensivo de poucos reprodutores e resultando na redução drástica do tamanho efetivo das populações. Contudo, vários estudos têm encontrado baixos coeficientes médios de endogamia, variando de 1,27\% a 3,81\% (Dias et al., 1994; Oliveira et al., 1999; Shimbo et al., 2000; Queiroz et al., 2000; Silva et al., 2001; Faria, 2002).

O baixo nível de endogamia relatado pode ser atribuído a diversas causas, como: animais fundadores de genealogia desconhecida (Shimbo et al., 2000; Queiroz et al., 2000); a prevenção de acasalamentos entre indivíduos aparentados (Oliveira et al., 1999; Dias et al., 1994); o parentesco entre os indivíduos não ser, em média, elevado (Alencar et al., 1981; Oliveira et al., 1999); o pequeno número de gerações consideradas na análise (Alencar et al., 1981; Oliveira et al., 1999).

Como conseqüências de taxas elevadas de endogamia, tem-se perda parcial do ganho genético obtido por seleção e redução do valor fenotípico médio, evidenciado, principalmente, pelos caracteres relacionados à capacidade reprodutiva ou eficiência fisiológica, fenômeno conhecido por depressão endogâmica. Entretanto, segundo Silva et al. (2001), a falta de programa de acasalamento eficiente e o fato de se trabalhar com rebanhos fechados têm sido fatores determinantes no avanço contínuo do nível de endogamia e do número de animais endogâmicos. Oliveira et al. (1999) ressaltam ainda a dificuldade de redução do coeficiente de endogamia quando a seleção é praticada em apenas uma característica.

Por outro lado, a endogamia tende a fixar alelos favoráveis e desfavoráveis em alguns locos. Espera-se, portanto, que ambas (a resposta total e a duração da resposta total) devam ser reduzidas, se a seleção for feita em uma população pequena com alta taxa de endogamia, e que a característica sob seleção seja determinada por um número finito de locos nãoligados agindo aditivamente. Teoricamente, quando a resposta à seleção diminui, a população pode estar no limite de seleção, que, ao ser alcançado, indica que todos os locos foram fixados.

Objetivou-se avaliar o comportamento do coeficiente de endogamia associado ao limite de seleção, considerando população-base selecionada sob diferentes métodos de seleção, diferentes tamanhos efetivos das populações e diferentes sistemas de acasalamentos.

R. Bras. Zootec., v.33, n.6, p.2017-2025, 2004 (Supl. 2)

\section{Material e Métodos}

A simulação dos dados foi feita por meio do programa GENESYS (Euclydes, 1996), que é constituído de quatorze programas escritos para o compilador FORTRAN. O programa permite a simulação de genomas de certa complexidade para os estudos de métodos de seleção, de testes de pressuposições matemáticas e de avaliações de novas metodologias de seleção.

Foi simulado um genoma com 500 locos quantitativos dialélicos distribuídos ao acaso em 30 pares de cromossomos autossômicos de tamanhos aleatórios com comprimento total de três mil centimorgans. O genoma simulado apresentou as seguintes propriedades: efeitos aditivos dos genes amostrados de uma distribuição normal; locos dialélicos sem interações não-aditivas e inexistência de cromossomos sexuais.

Os efeitos de ambiente não-controláveis foram simulados seguindo a distribuição normal e a variância que originasse a herdabilidade em estudo e as freqüências iniciais dos locos quantitativos foram amostradas de uma distribuição uniforme de média igual a 0,50 para ambos os sexos.

Foi simulada uma característica quantitativa de herdabilidade igual a 0,40, variância genética aditiva inicial igual a 450 e média correspondendo a 200 unidades fenotípicas, para a qual foi simulada uma populaçãobase constituída de mil indivíduos (500 machos e 500 fêmeas). Nessa população, desde a simulação dos gametas até a definição do sexo dos animais, foram usados processos inteiramente aleatórios.

A partir da população-base, foi simulada a população inicial, em que foram escolhidos e acasalados aleatoriamente 40 machos e 200 fêmeas, produzindo quatro descendentes por acasalamento, gerando 800 indivíduos, resultando em uma população com tamanho efetivo $\left(\mathrm{N}_{\mathrm{e}}\right)$ igual a 133,33 . A população inicial marca o início dos laços de parentesco entre os indivíduos, uma vez que, na população-base, os indivíduos foram originados de processos aleatórios, e não de segregações mendelianas.

Após a simulação da população inicial, foram formadas as populações de seleção, em que foram utilizados dois sistemas de acasalamentos (reprodutores acasalados aleatoriamente - RAA e exclusão de acasalamentos entre irmãos completos - EIC).

Na Tabela 1, são apresentados os valores de razão sexual, o número de fêmeas e de machos selecionados, a intensidade de seleção aplicada nas 
Tabela 1 - Valores de razão sexual (d), número de machos selecionados $\left(\mathrm{N}_{\mathrm{m}}\right)$, intensidade de seleção aplicada aos machos $\left(\mathrm{i}_{\mathrm{m}}\right)$, número de fêmeas selecionadas $\left(N_{f}\right)$, intensidade de seleção aplicada às fêmeas $\left(\mathrm{i}_{\mathrm{f}}\right)$ para os dois tamanhos efetivos das populações

Table 1 - Mating ratio levels (d), number of selected sires $(\mathrm{Nm})$, sire selection intensity (im), number of selected females (Nf), females selection intensity (if) for two effective population sizes

\begin{tabular}{ccccccc}
\hline População & $d=N_{f} / N_{m}$ & $N_{m}$ & $i_{m}$ & $N_{f}$ & $i_{f}$ & $N_{e}$ \\
\hline$N_{\mathrm{e} 1} 1$ & 20 & 10 & 2,33 & 200 & 0,80 & 38,09 \\
$\mathrm{~N}_{\mathrm{e} 2}$ & 08 & 25 & 1,96 & 200 & 0,80 & 88,88 \\
\hline
\end{tabular}

${ }^{1} \mathrm{~N}_{\mathrm{e} 1}=$ Tamanho efetivo um; ${ }^{2} \mathrm{~N}_{\mathrm{e} 2}=$ Tamanho efetivo dois.

fêmeas e nos machos e os dois tamanhos efetivos de populações simulados. Os tamanhos efetivos foram estabelecidos tomando-se por referência o estudo realizado por Faria (2002) sobre a estrutura genética das populações zebuínas brasileiras. Esse autor reportou tamanhos efetivos de 45, 24, 117, 41, 68, 120, 9 e 55 para as raças Gir, Gir mocho, Guzerá, Indubrasil, Nelore, Nelore mocho, Sindi e Tabapuã, respectivamente.

A partir da população inicial, empregaram-se dois esquemas de seleção, em que machos e fêmeas foram selecionados com base no fenótipo do indivíduo (SI) ou no valor genético predito pelo BLUP (melhor preditor linear não-viesado), com o uso do modelo animal, sendo consideradas as informações de pedigree das três últimas gerações. No modelo estatístico, foram incluídos os efeitos fixos de sexo e um efeito fixo qualquer, com 10 classes ou níveis, e os efeitos aleatórios de animal e de ambiente. O processo foi repetido 20 vezes, a fim de reduzir os efeitos de flutuação genética (Carneiro, 2002).

Foram consideradas três gerações distintas como gerações base, zero, três e sete, definidas por $\mathrm{G}_{0} \mathrm{~PB}$, $\mathrm{G}_{3} \mathrm{~PB}$ e $\mathrm{G}_{7} \mathrm{~PB}$, respectivamente. Na primeira, o processo de seleção foi conduzido por 17 gerações consecutivas e discretas, considerando-se todas as informações de pedigree desde a população-base verdadeira $\left(\mathrm{G}_{0} \mathrm{~PB}\right)$. Na segunda, procedeu-se à seleção por três gerações consecutivas e discretas, sendo a terceira geração, apesar de formada por indivíduos selecionados, considerada como geraçãobase $\left(G_{3} P B\right)$ e, a partir dela, o processo de seleção foi conduzido até a $13^{a}$ geração (Figura 1a). Na terceira geração-base simulada, a seleção foi conduzida por sete gerações consecutivas e discretas, de modo que a sétima geração foi considerada base $\left(\mathrm{G}_{7} \mathrm{~PB}\right)$ e selecionada até a $17^{\mathrm{a}}$ geração (Figura 1b). Os dois últimos procedimentos foram feitos para verificar o efeito da seleção ao se desconsiderar a genealogia nas gerações anteriores.

Para avaliar o efeito do uso de populações-base selecionadas ( $G_{3} P B$ e $\left.G_{7} P B\right)$, no decorrer das gerações, adotaram-se o coeficiente de endogamia médio, a percentagem de locos fixados favoravelmente e o limite de seleção.

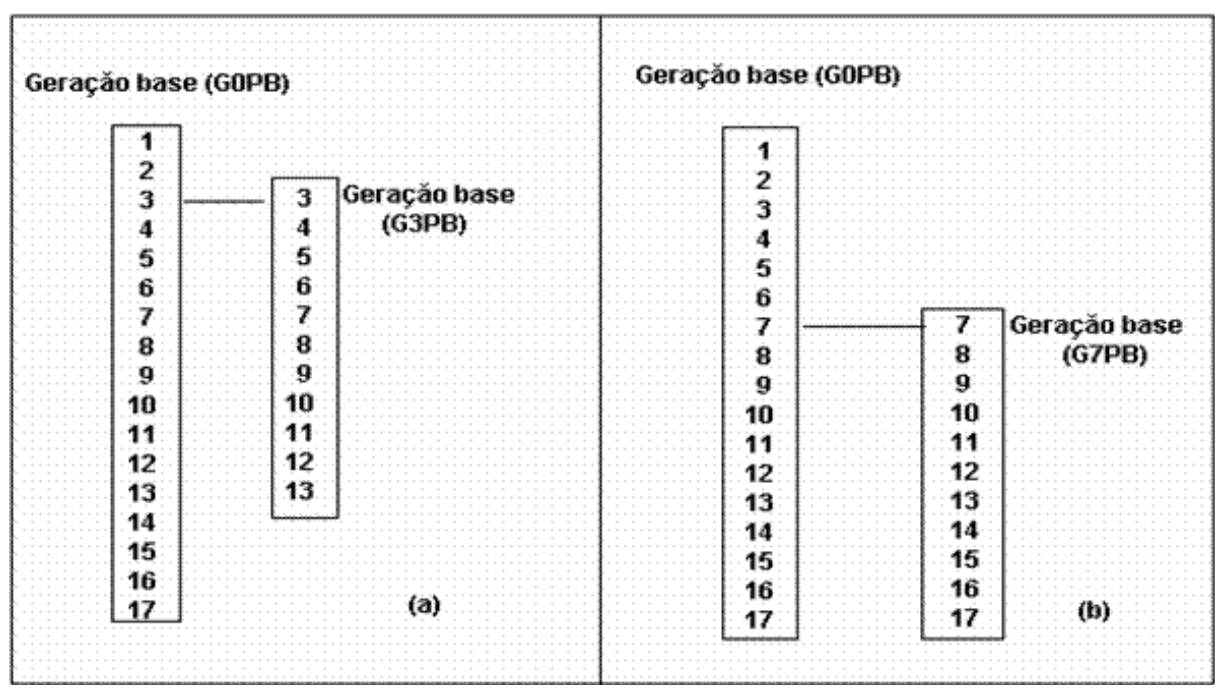

Figura 1 - Esquema da simulação das populações utilizando gerações-base distintas. Seleção da $1^{\mathrm{a}}$ à $17^{\mathrm{a}}$ geração $G_{0} P B(a, b)$; seleção da $3^{a}$ à $13^{a}$ geração $-G_{3} P B(a)$ e seleção da $7^{a}$ à $17^{a}$ geração $-G_{7} P B(b)$.

Figure 1 - Populations simulation scheme using distinct base generations. Selection on $1_{\text {th }}$ to $17_{\text {th }}$ generation $-G_{0} P B$ (a, b); selection on $3_{\text {th }}$ to $13_{\text {th }}$ generation $-G_{3} P B$ (a) and selection on $7_{\text {th }}$ to $17_{\text {th }}$ generation $-G_{7} P B$ (b).

R. Bras. Zootec., v.33, n.6, p.2017-2025, 2004 (Supl. 2) 
O limite de seleção foi definido de acordo com a probabilidade de fixação de alelos favoráveis e desfavoráveis. Alcança valores máximos quando ocorre a fixação de todos os alelos favoráveis e reduz quando apenas alelos desfavoráveis são fixados. A porcentagem de locos fixados, por sua vez, foi calculada em função do número total de locos quantitativos para cada característica.

\section{Resultados e Discussão}

Nas Tabelas 2 e 3, são apresentados os coeficientes médios de endogamia por geração de populações selecionadas com base nos valores genéticos preditos pelo BLUP para dois tamanhos efetivos de população $\left(\mathrm{N}_{\mathrm{e} 1}=38,09\right.$ e $\left.\mathrm{N}_{\mathrm{e} 2}=88,88\right)$, dois sistemas de acasalamento dos reprodutores selecionados (reprodutores acasalados aleatoriamente - RAA e exclusão de irmãos completos - EIC) e três gerações consideradas como populações-base (geração zero $G_{0} P B$, geração três - $G_{3} P B$ e geração sete $-G_{7} P B$ ). Nas Tabelas 4 e 5, estas informações são apresentadas para a seleção com base no fenótipo do indivíduo.

Em todos os esquemas simulados, verificou-se tendência crescente do coeficiente de endogamia no decorrer das gerações, como observado também por Dias et al. (1994) e Queiroz et al. (2000), em bovinos da raça Caracu e Gir, respectivamente. Os autores atribuíram esse comportamento à estrutura dos rebanhos, em que os animais fundadores são pouco aparentados ou de genealogia desconhecida e com o passar do tempo, mediante a ocorrência de acasalamentos dentro de rebanho, tornam-se endogâmicos. Alencar et al. (1981), em bovinos da raça Canchim, verificaram essa tendência, embora de maneira oscilatória.

Foram observadas pequenas diferenças, com relação à endogamia, entre os sistemas de acasalamentos, nas populações provenientes das três distintas gerações-base, para os dois tamanhos efetivos de população e métodos de seleção avaliados. Segundo Falconer (1987), o grau de parentesco entre os indivíduos depende do tamanho efetivo da população, sendo pares acasalados ao acaso mais proximamente aparentados em uma população pequena que em uma grande população. Portanto, esperar-se-ia que as populações em que os reprodutores foram acasalados aleatoriamente apresentassem maiores coeficientes médios de endogamia que as populações em que se excluíram acasalamentos entre irmãos completos, principalmente nas de menor tamanho efetivo. Entretanto, Falconer \& Mackay (1996) relataram que a exclusão de acasalamentos entre indivíduos proximamente relacionados não proporciona grande diferença na taxa de endogamia. A progênie resultante do acasalamento entre irmãos completos possui maior coeficiente de endogamia que aquelas provenientes de acasalamentos menos relacionados, podendo aumentar o coeficiente de endogamia da população em qualquer geração. Todavia, a maior taxa de endogamia gerada por este tipo de acasalamento não é permanente e, sob acasalamento ao acaso, é diluída nas gerações seguintes como conseqüência de acasalamentos entre indivíduos menos aparentados. Assim, a exclusão de acasalamentos entre irmãos pode reduzir o coeficiente médio de endogamia, mas não tem efeito expressivo sobre seu acúmulo.

As populações de menor tamanho efetivo $\left(\mathrm{N}_{\mathrm{e} 1}\right)$ apresentaram os maiores coeficientes de endogamia, em razão da maior probabilidade de acasalamentos entre os indivíduos aparentados nessas populações, em comparação com as de maior tamanho efetivo. Esse resultado está de acordo com o relatado por Van Der Werf \& De Boer (1990). Outro fator seria a maior intensidade de seleção praticada nas populações de $\mathrm{N}_{\mathrm{e} 1}$. Segundo Silva et al. (2001), a intensa utilização de touros com valores genéticos mais elevados para uma característica de importância econômica tende a aumentar os laços genéticos entre os reprodutores.

Quando os animais foram selecionados por meio de valores genéticos preditos pelo BLUP, observou-se coeficiente médio de endogamia abaixo de $5 \%$ nas três e cinco primeiras gerações no $\mathrm{N}_{\mathrm{e} 1}$ e $\mathrm{N}_{\mathrm{e} 2}$, respectivamente, nas populações $\mathrm{G}_{3} \mathrm{~PB}$ e nas quatro primeiras gerações de seleção, para os dois tamanhos efetivos, nas populações que consideraram a geração sete como base (Tabelas 2 e 3). Já nas populações em que se computaram as informações de parentesco desde a geração-base verdadeira $\left(\mathrm{G}_{0} \mathrm{~PB}\right)$ os coeficientes de endogamia alcançaram valores superiores a $8,5 \%$ no $\mathrm{N}_{\mathrm{e} 1}$ e 5,2\% no $\mathrm{N}_{\mathrm{e} 2}$ (Tabela 2) e 20 e $12 \%$ nas populações de $\mathrm{N}_{\mathrm{e} 1}$ e $\mathrm{N}_{\mathrm{e} 2}$, respectivamente (Tabela 3).

Os valores dos coeficientes médios de endogamia nas populações $\mathrm{G}_{3} \mathrm{~PB}$ e $\mathrm{G}_{7} \mathrm{~PB}$, comparados com $\mathrm{G}_{0} \mathrm{~PB}$, foram subestimados em todas as gerações, como esperado.

Nas populações em que os animais foram selecionados por meio do fenótipo individual, observou-se, nas populações $\mathrm{G}_{3} \mathrm{~PB}$, que até a $7^{\mathrm{a}}$ e $11^{\mathrm{a}}$ gerações no $\mathrm{N}_{\mathrm{e} 1}$ e $\mathrm{N}_{\mathrm{e} 2}$, respectivamente, os coeficientes 
Tabela 2 - Coeficiente médio de endogamia da $3^{\mathrm{a}}$ à $13^{\mathrm{a}}$ geração, em porcentagem, utilizando BLUP com RAA e EIC, para $\mathrm{N}_{\mathrm{e} 1}$ e $\mathrm{N}_{\mathrm{e} 2}$, considerando a geração zero $\left(G_{0} P B\right)$ e a geração três $\left(G_{3} P B\right)$ como populações-base

Table 2 - Average inbreeding coefficient from $3^{a}$ to $13^{a}$ generation, in percentage, using BLUP with RAA and EIC, for $\mathrm{Ne}_{1}$ and $\mathrm{Ne}_{2}$, considering the zero generation $\left(G_{0} P B\right)$ and the three generation $\left(G_{3} P B\right)$ as base population

\begin{tabular}{|c|c|c|c|c|c|c|c|c|}
\hline \multirow[b]{4}{*}{ Geração } & \multicolumn{8}{|c|}{ Endogamia (\%) } \\
\hline & \multicolumn{4}{|c|}{$\mathrm{G}_{0} \mathrm{~PB}$} & \multicolumn{4}{|c|}{$\mathrm{G}_{3} \mathrm{~PB}$} \\
\hline & \multicolumn{2}{|c|}{$\mathrm{N}_{\mathrm{e} 1}=38,09$} & \multicolumn{2}{|c|}{$\mathrm{N}_{\mathrm{e} 2}=88,88$} & \multicolumn{2}{|c|}{$\mathrm{N}_{\mathrm{e} 1}=38,09$} & \multicolumn{2}{|c|}{$\mathrm{N}_{\mathrm{e} 2}=88,88$} \\
\hline & RAA $^{1}$ & $\mathrm{EIC}^{2}$ & RAA & EIC & RAA & EIC & RAA & EIC \\
\hline 3 & 0,087 & 0,084 & 0,052 & 0,052 & 0,000 & 0,000 & 0,000 & 0,000 \\
\hline 4 & 0,126 & 0,115 & 0,074 & 0,071 & 0,000 & 0,000 & 0,000 & 0,000 \\
\hline 5 & 0,163 & 0,146 & 0,092 & 0,091 & 0,025 & 0,017 & 0,012 & 0,009 \\
\hline 6 & 0,194 & 0,173 & 0,113 & 0,109 & 0,057 & 0,052 & 0,029 & 0,029 \\
\hline 7 & 0,220 & 0,207 & 0,131 & 0,128 & 0,094 & 0,087 & 0,047 & 0,050 \\
\hline 8 & 0,247 & 0,239 & 0,146 & 0,146 & 0,128 & 0,123 & 0,064 & 0,071 \\
\hline 9 & 0,275 & 0,268 & 0,160 & 0,164 & 0,165 & 0,150 & 0,082 & 0,091 \\
\hline 10 & 0,300 & 0,292 & 0,174 & 0,179 & 0,192 & 0,180 & 0,101 & 0,111 \\
\hline 11 & 0,321 & 0,320 & 0,189 & 0,193 & 0,219 & 0,212 & 0,124 & 0,128 \\
\hline 12 & 0,349 & 0,340 & 0,208 & 0,210 & 0,248 & 0,243 & 0,140 & 0,147 \\
\hline 13 & 0,377 & 0,360 & 0,228 & 0,226 & 0,272 & 0,269 & 0,157 & 0,164 \\
\hline
\end{tabular}

${ }^{1} \mathrm{RAA}=$ reprodutores acasalados aleatoriamente; ${ }^{2} \mathrm{EIC}=$ excluindo acasalamentos entre irmãos completos.

${ }^{1} R A A=$ random mating; ${ }^{2} E I C=$ exclusion of mating between full sibs.

Tabela 3 - Coeficiente médio de endogamia da $7^{\mathrm{a}}$ a $17^{\mathrm{a}}$ geração, em porcentagem, utilizando BLUP com RAA e EIC, para $\mathrm{N}_{\mathrm{e} 1}$ e $\mathrm{N}_{\mathrm{e} 2}$, considerando a geração zero $\left(G_{0} P B\right)$ e a geração sete $\left(G_{7} P B\right)$ como populações-base

Table 3 - Average inbreeding coefficient from $7^{a}$ to $17^{a}$ generation, in percentage, using BLUP with RAA and EIC, for $\mathrm{Ne}_{1}$ and $\mathrm{Ne}_{2}$, considering the zero generation $\left(\mathrm{G}_{0} P B\right)$ and the seven generation $\left(G_{7} P B\right)$ as base population

\begin{tabular}{|c|c|c|c|c|c|c|c|c|}
\hline \multirow[b]{4}{*}{ Geração } & \multicolumn{8}{|c|}{ Endogamia (\%) } \\
\hline & \multicolumn{4}{|c|}{$\mathrm{G}_{0} \mathrm{~PB}$} & \multicolumn{4}{|c|}{$\mathrm{G}_{7} \mathrm{~PB}$} \\
\hline & \multicolumn{2}{|c|}{$\mathrm{N}_{\mathrm{e} 1}=38,09$} & \multicolumn{2}{|c|}{$\mathrm{N}_{\mathrm{e} 2}=88,88$} & \multicolumn{2}{|c|}{$\mathrm{N}_{\mathrm{e} 1}=38,09$} & \multicolumn{2}{|c|}{$\mathrm{N}_{\mathrm{e} 2}=88,88$} \\
\hline & $\mathrm{RAA}^{1}$ & $\mathrm{EIC}^{2}$ & RAA & EIC & RAA & EIC & RAA & EIC \\
\hline 7 & 0,220 & 0,207 & 0,131 & 0,128 & 0,000 & 0,000 & 0,000 & 0,000 \\
\hline 8 & 0,240 & 0,239 & 0,146 & 0,146 & 0,000 & 0,000 & 0,000 & 0,000 \\
\hline 9 & 0,270 & 0,268 & 0,160 & 0,164 & 0,021 & 0,021 & 0,013 & 0,011 \\
\hline 10 & 0,299 & 0,292 & 0,174 & 0,179 & 0,048 & 0,048 & 0,034 & 0,033 \\
\hline 11 & 0,321 & 0,320 & 0,189 & 0,193 & 0,076 & 0,081 & 0,060 & 0,052 \\
\hline 12 & 0,349 & 0,343 & 0,208 & 0,210 & 0,104 & 0,113 & 0,081 & 0,072 \\
\hline 13 & 0,377 & 0,360 & 0,228 & 0,226 & 0,136 & 0,149 & 0,103 & 0,088 \\
\hline 14 & 0,404 & 0,383 & 0,250 & 0,243 & 0,166 & 0,181 & 0,126 & 0,103 \\
\hline 15 & 0,423 & 0,408 & 0,268 & 0,259 & 0,191 & 0,209 & 0,142 & 0,118 \\
\hline 16 & 0,444 & 0,429 & 0,282 & 0,274 & 0,214 & 0,238 & 0,158 & 0,132 \\
\hline 17 & 0,461 & 0,452 & 0,298 & 0,288 & 0,242 & 0,260 & 0,171 & 0,150 \\
\hline
\end{tabular}

de endogamia se mantiveram abaixo de $5 \%$ (Tabela 4). Nas populações $\mathrm{G}_{7} \mathrm{~PB}$, por sua vez, verificou-se coeficiente de endogamia abaixo de $5 \%$ até a $11^{\text {a }}$ geração para $\mathrm{N}_{\mathrm{e} 1}$ e até a $15^{\mathrm{a}}$ geração para o $\mathrm{N}_{\mathrm{e} 2}$

\footnotetext{
R. Bras. Zootec., v.33, n.6, p.2017-2025, 2004 (Supl. 2)
}

(Tabela 5). Contudo, na $\mathrm{G}_{0} \mathrm{~PB}$, foram observados valores abaixo de $5 \%$ da $3^{\mathrm{a}}$ à $4^{\mathrm{a}}$ geração no $\mathrm{N}_{\mathrm{e} 1}$, da $3^{\mathrm{a}}$ à $8^{\mathrm{a}}$ geração no $\mathrm{N}_{\mathrm{e} 2}$ (Tabela 4) e apenas nas gerações sete e oito no $\mathrm{N}_{\mathrm{e} 2}$ (Tabela 5). 
As populações $G_{3} P B$ e $G_{7} P B$, selecionadas com base no fenótipo individual, também apresentaram valores dos coeficientes médios de endogamia subestimados, quando comparados com os valores obtidos nas populações $G_{0} P B$. Contudo, o viés nas estimativas foi menor quando comparado com as populações selecionadas com base no BLUP.

Tabela 4 - Coeficiente médio de endogamia da $3^{\mathrm{a}}$ a $13^{\mathrm{a}}$ geração, em percentagem, utilizando seleção com base no fenótipo individual com RAA e EIC, para $\mathrm{N}_{\mathrm{e}}$ e $N_{e 2}$, considerando a geração zero $\left(G_{0} P B\right)$ e a geração três $\left(G_{3} P B\right)$ como populações-base

Table 4 - Average inbreeding coefficient from $3^{a}$ to $13^{a}$ generation, in percentage, using individual selection with RAA and EIC, for $\mathrm{Ne}_{1}$ and $\mathrm{Ne}_{2}$, considering the zero generation $\left(G_{0} P B\right)$ and the three generation $\left(G_{3} P B\right)$ as base population

\begin{tabular}{|c|c|c|c|c|c|c|c|c|}
\hline \multirow[b]{4}{*}{ Geração } & \multicolumn{8}{|c|}{ Endogamia (\%) } \\
\hline & \multicolumn{4}{|c|}{$\mathrm{G}_{0} \mathrm{~PB}$} & \multicolumn{4}{|c|}{$\mathrm{G}_{3} \mathrm{~PB}$} \\
\hline & \multicolumn{2}{|c|}{$\mathrm{N}_{\mathrm{e} 1}=38,09$} & \multicolumn{2}{|c|}{$\mathrm{N}_{\mathrm{e} 2}=88,88$} & \multicolumn{2}{|c|}{$\mathrm{N}_{\mathrm{e} 1}=38,09$} & \multicolumn{2}{|c|}{$\mathrm{N}_{\mathrm{e} 2}=88,88$} \\
\hline & RAA $^{1}$ & $\mathrm{EIC}^{2}$ & RAA & EIC & RAA & EIC & RAA & EIC \\
\hline 3 & 0,034 & 0,034 & 0,015 & 0,015 & 0,000 & 0,000 & 0,000 & 0,000 \\
\hline 4 & 0,052 & 0,049 & 0,022 & 0,022 & 0,000 & 0,000 & 0,000 & 0,000 \\
\hline 5 & 0,068 & 0,064 & 0,030 & 0,028 & 0,015 & 0,014 & 0,005 & 0,005 \\
\hline 6 & 0,082 & 0,079 & 0,035 & 0,034 & 0,028 & 0,027 & 0,013 & 0,011 \\
\hline 7 & 0,097 & 0,092 & 0,042 & 0,041 & 0,042 & 0,040 & 0,019 & 0,018 \\
\hline 8 & 0,110 & 0,106 & 0,048 & 0,046 & 0,056 & 0,056 & 0,024 & 0,023 \\
\hline 9 & 0,127 & 0,119 & 0,053 & 0,052 & 0,070 & 0,071 & 0,032 & 0,029 \\
\hline 10 & 0,143 & 0,131 & 0,059 & 0,058 & 0,085 & 0,085 & 0,038 & 0,035 \\
\hline 11 & 0,154 & 0,144 & 0,066 & 0,065 & 0,101 & 0,102 & 0,045 & 0,042 \\
\hline 12 & 0,169 & 0,157 & 0,072 & 0,070 & 0,111 & 0,117 & 0,052 & 0,048 \\
\hline 13 & 0,182 & 0,169 & 0,078 & 0,077 & 0,129 & 0,132 & 0,085 & 0,053 \\
\hline
\end{tabular}

${ }^{1} \mathrm{RAA}=$ reprodutores acasalados aleatoriamente; ${ }^{2} \mathrm{EIC}=$ excluindo acasalamentos entre irmãos completos

${ }^{1} R A A=$ random mating; ${ }^{2} E I C=$ exclusion of mating between full sibs

Tabela 5 - Coeficiente médio de endogamia da $7^{\mathrm{a}}$ a $17^{\mathrm{a}}$ geração, em percentagem, utilizando seleção com base no fenótipo individual com RAA e EIC, para $\mathrm{N}_{\mathrm{e} 1}$ e $N_{e 2}$, considerando a geração zero $\left(G_{0} P B\right)$ e a geração sete $\left(G_{7} P B\right)$ como populações-base

Table 5 - Average inbreeding coefficient from $7^{a}$ to $17^{a}$ generation, in percentage, using individual selection with RAA and EIC, for $\mathrm{Ne}_{1}$ and $\mathrm{Ne}_{2}$, considering the zero generation $\left(G_{0} P B\right)$ and the seven generation $\left(G_{7} P B\right)$ as base population

\begin{tabular}{|c|c|c|c|c|c|c|c|c|}
\hline \multirow[b]{3}{*}{ Geração } & \multicolumn{4}{|c|}{$\mathrm{G}_{0} \mathrm{~PB}$} & \multicolumn{4}{|c|}{$\mathrm{G}_{7} \mathrm{~PB}$} \\
\hline & \multicolumn{2}{|c|}{$\mathrm{N}_{\mathrm{e} 1}=38,09$} & \multicolumn{2}{|c|}{$\mathrm{N}_{\mathrm{e} 2}=88,88$} & \multicolumn{2}{|c|}{$\mathrm{N}_{\mathrm{e} 1}=38,09$} & \multicolumn{2}{|c|}{$\mathrm{N}_{\mathrm{e} 2}=88,88$} \\
\hline & $\mathrm{RAA}^{1}$ & $\mathrm{EIC}^{2}$ & RAA & EIC & RAA & EIC & RAA & EIC \\
\hline 7 & 0,097 & 0,092 & 0,042 & 0,041 & 0,000 & 0,000 & 0,000 & 0,000 \\
\hline 8 & 0,110 & 0,106 & 0,048 & 0,046 & 0,000 & 0,000 & 0,000 & 0,000 \\
\hline 9 & 0,127 & 0,119 & 0,053 & 0,052 & 0,014 & 0,013 & 0,006 & 0,005 \\
\hline 10 & 0,143 & 0,131 & 0,059 & 0,058 & 0,027 & 0,029 & 0,012 & 0,012 \\
\hline 11 & 0,154 & 0,144 & 0,066 & 0,065 & 0,042 & 0,043 & 0,018 & 0,018 \\
\hline 12 & 0,169 & 0,157 & 0,072 & 0,070 & 0,058 & 0,057 & 0,025 & 0,025 \\
\hline 13 & 0,182 & 0,169 & 0,078 & 0,077 & 0,073 & 0,071 & 0,030 & 0,031 \\
\hline 14 & 0,196 & 0,183 & 0,085 & 0,082 & 0,088 & 0,088 & 0,038 & 0,037 \\
\hline 15 & 0,209 & 0,194 & 0,090 & 0,087 & 0,100 & 0,101 & 0,043 & 0,044 \\
\hline 16 & 0,223 & 0,205 & 0,095 & 0,094 & 0,113 & 0,113 & 0,051 & 0,051 \\
\hline 17 & 0,234 & 0,217 & 0,102 & 0,098 & 0,126 & 0,127 & 0,056 & 0,057 \\
\hline
\end{tabular}

${ }^{1} \mathrm{RAA}=$ reprodutores acasalados aleatoriamente; ${ }^{2} \mathrm{EIC}=$ excluindo acasalamentos entre irmãos completos

${ }^{1} R A A=$ random mating; ${ }^{2} E I C=$ exclusion of mating between full sibs

R. Bras. Zootec., v.33, n.6, p.2017-2025, 2004 (Supl. 2) 
Tanto na seleção com base no fenótipo individual quanto na seleção com base nos valores genéticos preditos pelo BLUP, as populações $\mathrm{G}_{7} \mathrm{~PB}$ foram as que apresentaram maiores subestimativas dos valores dos coeficientes médios de endogamia em relação às populações que consideraram a $\mathrm{G}_{3} \mathrm{~PB}$. Isso ocorreu porque na G7PB desconsiderou-se o conhecimento da genealogia de um número maior de gerações.

O baixo coeficiente de endogamia encontrado nas três primeiras gerações, principalmente, quando não se considerou a informação de pedigree até a geração zero em populações de $\mathrm{N}_{\mathrm{e} 2}$, selecionadas com base no BLUP e no fenótipo individual, está de acordo com os reportados na literatura. Alencar et al. (1981) relataram coeficiente médio de endogamia da população de $1,84 \%$ na raça Canchim; Dias et al. (1994), de 2,23\% na raça Caracu; Shimbo et al. (2000) e Faria (2002), de 3,81 e 2,13\%, respectivamente, na raça Nelore; e Faria (2002), de 0,98; 1,75; 2,28; 3,06; 3,44 e 1,58\%, nas raças Nelore mocho, Guzerá, Gir, Gir mocho, Indubrasil, Sindi e Tabapuã, respectivamente.

Alguns autores ressaltam que o baixo coeficiente de endogamia encontrado pode ser atribuído ao pequeno número de gerações em análise, geralmente em torno de quatro, e ao fato de os animais fundadores serem de genealogia desconhecida, situação semelhante à das populações simuladas $\mathrm{G}_{3} \mathrm{~PB}_{\mathrm{P}} \mathrm{G}_{7} \mathrm{~PB}$.

Admitindo que a população-base (coeficiente de endogamia igual a zero) é composta, sobretudo, por animais degenealogia desconhecida, Queiroz et al.(2000) ressaltam que o melhor conhecimento da genealogia possibilita cálculo mais acurado do coeficiente de endogamia, com conseqüente aumento de seus valores.

Quanto aos tamanhos efetivos, as maiores subestimativas dos coeficientes de endogamia, quando não se considera a geração zero como base, foram observadas nas populações de menor tamanho $\left(\mathrm{N}_{\mathrm{e} 1}\right)$, nos dois sistemas de acasalamentos e métodos de seleção. Entretanto, vale ressaltar que as diferenças foram de menor magnitude nas populações selecionadas com base no fenótipo do indivíduo que nas populações selecionadas com base nos valores genéticos preditos pelo BLUP.

Considerando as $\mathrm{G}_{3} \mathrm{~PB}$ e $\mathrm{G}_{7} \mathrm{~PB}$, as diferenças no aumento da endogamia entre os tamanhos efetivos foram bem menores que os observados nas populações $\mathrm{G}_{0} \mathrm{~PB}$, especialmente nas populações que utilizaram a $G_{7} P B$. Isso resultou do maior coeficiente de endogamia presente na $3^{\mathrm{a}}$ e $7^{\mathrm{a}}$ gerações de seleção, em que se tinham informações de pedigree desde a geração zero. Como estes coeficientes são ainda maiores nas populações de $\mathrm{N}_{\mathrm{e} 1}$, quando se quantifica a diferença entre os tamanhos efetivos, observam-se maiores diferenças para as populações em que se consideram a $G_{0} P B$, pois o coeficiente de endogamia para os dois tamanhos efetivos na $3^{\mathrm{a}}$ e $7^{\mathrm{a}}$ geração nas populações $\mathrm{G}_{3} \mathrm{~PB}$ e $\mathrm{G}_{7} \mathrm{~PB}$, respectivamente, é considerado igual a zero.

De maneira geral, as populações selecionadas pelo BLUP foram as que apresentaram maiores coeficientes de endogamia, corroborando os resultados encontrados por Cunha et al. (2003) e Quinton et al. (1992). A seleção baseada em estimativas de valores genéticos por meio do BLUP com o modelo animal geralmente conduz a incrementos na taxa de endogamia, pois animais aparentados possuem valores genéticos similares (Caballero et al., 1996; Queiroz et al., 2000).

A endogamia provoca redução no mérito genético individual nas características produtivas, possivelmente, graças à ocorrência de fixação de alelos desfavoráveis. Logicamente, o efeito depressivo é relativamente menor em baixos níveis de endogamia. Portanto, o monitoramento do parentesco entre os animais selecionados para a reprodução é indicado para manutenção ou redução da endogamia da população.

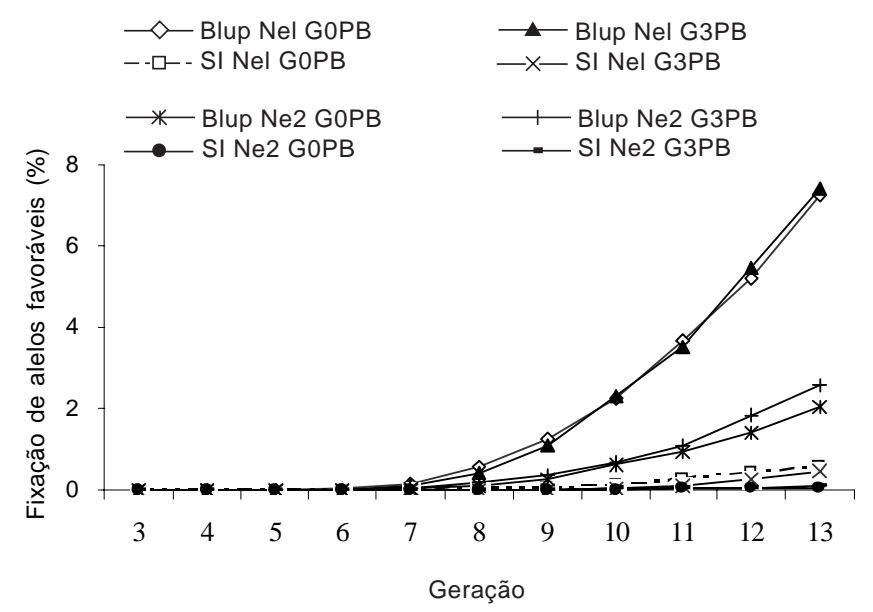

Figura 2 - Valores, em percentagem, de alelos favoráveis fixados obtidos pela seleção baseada no BLUP e fenótipo individual (SI), para populações de $\mathrm{N}_{\mathrm{e} 1}$ e $\mathrm{N}_{\mathrm{e} 2}$, considerando $\mathrm{G}_{0} P B$ e $\mathrm{G}_{3} \mathrm{~PB}$, com exclusão de irmãos completos.

Figure 2 - Values, in percentage, fixation of favorable alleles obtained by selection based BLUP and individual phenotype (SI), for populations of $N_{e 1}$ and $N_{e 2}$, considering $G_{0} P B$ and $G_{3} P B$, with exclusion of full sibs.

R. Bras. Zootec., v.33, n.6, p.2017-2025, 2004 (Supl. 2) 
Nas Figuras 2 e 3, constam os valores, em percentagem, de alelos favoráveis fixados para as populações $G_{0} P B$ e $G_{3} P B$ e, $G_{0} P B$ e $G_{7} P B$, respectivamente, obtidos pela seleção baseada no BLUP e SI, excluindo acasalamentos entre irmãos completos (EIC), nas populações de $\mathrm{N}_{\mathrm{e} 1}$ e $\mathrm{N}_{\mathrm{e} 2}$.

À medida que aumentou o coeficiente médio de endogamia ao longo das gerações, verificou-se progresso nas taxas de fixação de alelos favoráveis.

As populações selecionadas pelo BLUP foram as que apresentaram os maiores coeficientes médios de endogamia e as maiores percentagens de fixação de alelos favoráveis. Caballero et al. (1996), Queiroz et al. (2000)eCunha etal.(2003)reportaram que, naspopulações selecionadas pelo BLUP, existe melhor acurácia de seleção, favorecendo a fixação de alelos favoráveis, além de aumento do peso atribuído à informação de família, que acabam por elevar a taxa de endogamia.

Na comparação dos dois tamanhos efetivos, observou-se que, na população de $\mathrm{N}_{\mathrm{e} 2}$, houve menor percentagem de fixação de alelos favoráveis, em razão do menor acúmulo de endogamia e da menor intensidade de seleção praticada nessas populações, sendo a maior diferença, quanto ao tamanho efetivo, mais evidente nas populações selecionadas pelo BLUP.

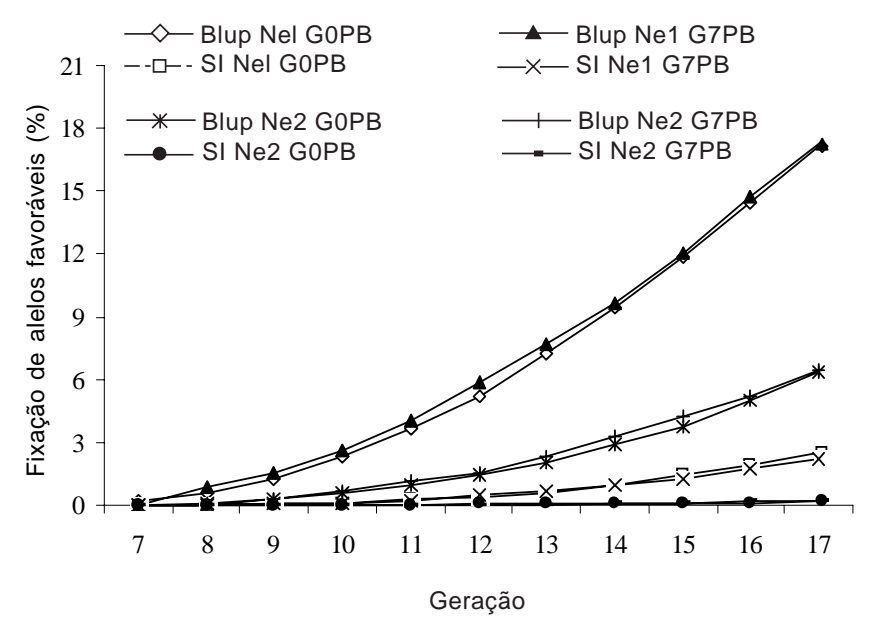

Figura 3 - Valores, em percentagem, de alelos favoráveis fixados obtidos pela seleção baseada no BLUP e fenótipo individual (SI), para populações de $\mathrm{N}_{\mathrm{e} 1}$ e $\mathrm{N}_{\mathrm{e} 2}$, considerando $\mathrm{G}_{0} P B$ e $\mathrm{G}_{7} \mathrm{~PB}$, com exclusão de irmãos completos.

Figure 3 - Values, in percentage, fixation of favorable alleles obtained by selection based BLUP and individual phenotype (SI), for populations of $N_{e 1}$ and $N_{e 2}$, considering $G_{0} P B$ and $G_{7} P B$, with exclusion of full sibs.
Não foi observada diferença de grande magnitude na comparação das populações $\mathrm{G}_{0} \mathrm{~PB}$ e $\mathrm{G}_{3} \mathrm{~PB}$ e, $\mathrm{G}_{0} \mathrm{~PB}$ e $\mathrm{G}_{7} \mathrm{~PB}$, em relação aos tamanhos efetivos e métodos de seleção.

Na Figura 4, são apresentados os valores de limite de seleção utilizando $G_{0} P B$ e $G_{3} P B$. Do início até a $6^{\text {a }}$ geração, não foram observados decréscimos nos valores do limite de seleção para os dois métodos de seleção. A partir desta geração, houve clara redução no limite de seleção nas populações selecionadas com base no BLUP $\left(\mathrm{N}_{\mathrm{e} 1}\right)$. Os valores de limite de seleção utilizando $G_{0} P B$ e $G_{7} P B$ podem ser observados na Figura 5. Decréscimos nas populações selecionadas com base no BLUP ocorreram a partir da $7^{\mathrm{a}}$ geração nos dois tamanhos efetivos.

Ressalta-se que as populações com maior tamanho efetivo foram as que apresentaram menores reduções nos valores do limite de seleção.

Nas populações mantidas sob seleção individual $\operatorname{com~} \mathrm{N}_{\mathrm{e} 1}$, houve pequena redução do limite de seleção a partir da $9^{\mathrm{a}}$ e $12^{\mathrm{a}}$ gerações nas populações $\mathrm{G}_{0} \mathrm{~PB}$ e $\mathrm{G}_{3} \mathrm{~PB}$ e, $\mathrm{G}_{0} \mathrm{~PB}$ e $\mathrm{G}_{7} \mathrm{~PB}$, respectivamente. No $\mathrm{N}_{\mathrm{e} 2}$, praticamente não houve redução nos valores do limite de seleção em todas as condições consideradas.

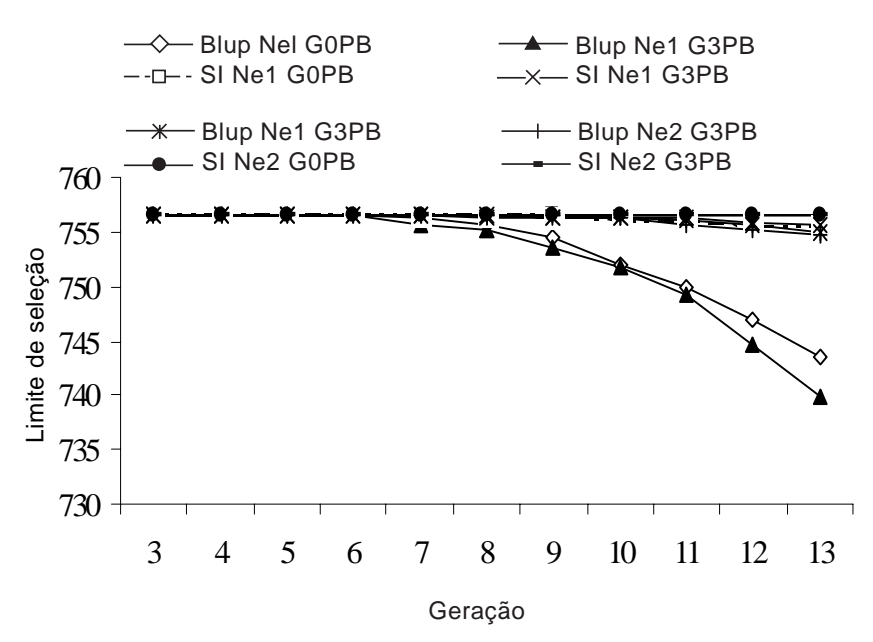

Figura 4 - Valores do limite de seleção obtidos pela seleção baseada no BLUP e seleção com base no fenótipo individual (SI), para populações de $\mathrm{N}_{\mathrm{e} 1}$ e $\mathrm{N}_{\mathrm{e} 2}$, considerando $\mathrm{G}_{0} P B$ e $\mathrm{G}_{3} \mathrm{~PB}$, com exclusão de irmãos completos.

Figure 4 - Selection limit value obtained by selection based $B L U P$ and individual phenotype (SI), for populations of $N_{e 1}$ and $N_{e 2}$, considering $G_{0} P B$ and $G_{3} P B$, with exclusion of full sibs. 


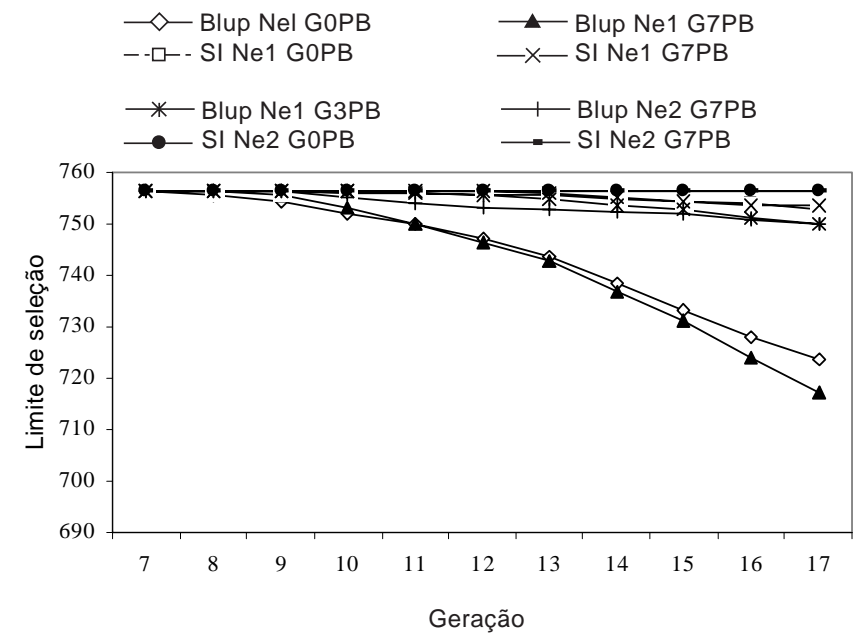

Figura 5 - Valores do limite de seleção obtidos pela seleção baseada BLUP e seleção com base no fenótipo individual (SI), para populações de $\mathrm{N}_{\mathrm{e} 1}$ e $\mathrm{N}_{\mathrm{e} 2}$, considerando $\mathrm{G}_{0} \mathrm{~PB}$ e $\mathrm{G}_{7} \mathrm{~PB}$, com exclusão de irmãos completos.

Figure 5 - Selection limit value obtained by selection based $B L U P$ and individual phenotype (SI), for populations of $N_{e 1}$ and $N_{e 2}$, considering $G_{0} P B$ and $G_{7} P B$, with exclusion of full sibs.

Na comparação das populações $\mathrm{G}_{3} \mathrm{~PB}$ e $\mathrm{G}_{7} \mathrm{~PB}$ com as $G_{0} P B$, não foi observada diferença de grande magnitude quanto ao limite de seleção.

\section{Conclusões}

As populações de menor tamanho efetivo apresentaram os maiores coeficientes médios de endogamia, a maior fixação de alelos favoráveis e a maior redução do limite de seleção, principalmente quando selecionadas com base nos valores genéticos preditos pelo BLUP.

A não-utilização da população-base verdadeira $\left(\mathrm{G}_{0} \mathrm{~PB}\right)$ subestima os coeficientes de endogamia e, quanto maior o número de gerações desprezadas, maior o viés. Contudo, mesmo apresentando baixos valores de endogamia, essas populações continuam a fixar alelos favoráveis e a alcançar o limite de seleção como as populações que utilizam a população-base verdadeira.

A exclusão de acasalamentos entre irmãos completos não evita o acúmulo de endogamia a médio e longo prazos.

\section{Literatura Citada}

ALENCAR, M.M.; SILVA, A.H.G.; BARBOSA, P.F. Efeitos da consangüinidade sobre os pesos ao nascimento e à desmama de bezerros da raça Canchim. Revista Brasileira de Zootecnia, v.10, n.1, p.157-172, 1981.

CABALLERO, A.; SANTIAGO, E.; TORO, M.A. Systems of mating to reduce inbreeding in selected populations. Animal science, v.62, p.431-442, 1996.

CARNEIRO, P.L. Oscilação genética e comparação de métodos de seleção tradicionais e associados a marcadores moleculares. Viçosa, MG: Universidade Federal de Viçosa, 2002. 104p. Tese (Doutorado em Genética e Melhoramento) - Universidade Federal de Viçosa, 2002.

CUNHA, E.E.; EUCLYDES, R.F.; TORRES, R.A. et at. Efeito de tipos de acasalamentos e razões sexuais na seleção baseada no BLUP. Revista Brasileira de Zootecnia, v.32, n.6, p.1297-1303, 2003.

DIAS, A.S.C.; QUEIROZ, S.A.; ALBUQUERQUE, L.G. Efeito da endogamiaem características reprodutivas debovinos daraçaCaracu. Revista Brasileira de Zootecnia, v.23, n.2, p.157-164, 1994.

EUCLYDES, R.F. Uso do sistema para simulação Genesys na avaliação de métodos de seleção clássicos e associados a marcadores moleculares. Viçosa, MG: Universidade Federal de Viçosa, 1996. 150p. Tese (Doutorado em Genética e Melhoramento) - Universidade Federal de Viçosa, 1996.

FALCONER, D.S. Introdução à genética quantitativa. 1.ed. Viçosa, MG: Universidade Federal de Viçosa, 1987. 279p.

FALCONER, D.S.; MACKAY, T.F.C. Introduction to quantitative genetics. 4.ed. Londom: Longman, 1996. 464p.

FARIA, F.J.C. Estrutura genética das populações zebuínas brasileiras registradas. Belo Horizonte: Universidade Federal de Minas Gerais, 2002. 243p. Tese (Doutorado em Melhoramento Animal) - Universidade Federal de Minas Gerais, 2002.

OLIVEIRA, J.A.; BASTOS, J.F.P.; TONHATI, H. Endogamia em um rebanho da raça Guzerá. Revista Brasileira de Zootecnia, v.28, n.4, p.721-728, 1999.

QUEIROZ, S.A.; ALBUQUERQUE, L.G.; LANZONI, N.A. Efeito da endogamia sobre características de crescimento de bovinos da raça Gir no Brasil. Revista Brasileira de Zootecnia, v.29, n.4, p.1014-1019, 2000.

QUINTON, M.; SMITH, C.; GODDARD, M.E. Comparison of selection methods at the same level of inbreeding. Journal of Animal Science, v.70, p.1060-1067, 1992.

SHIMBO, M.V.; FERRAZ, J.B.S.; ELER, J.P. et al. Influência da endogamia sobre característica de desempenho em bovinos da raça Nelore. In: SIMPÓSIO NACIONAL DA SOCIEDADE BRASILEIRA DE MELHORAMENTO ANIMAL, 3., 2000, Belo Horizonte. Anais... Belo Horizonte: Sociedade Brasileira de Melhoramento Animal, 2000. p.388-390.

SILVA, M.V.G.B.; FERREIRA, W.J.F.; COBUCI, J.A. et al. Efeito da endogamia sobre características produtivas e reprodutivas de bovinos do ecótipo Mantiqueira. Revista Brasileira de Zootecnia, v.30, n.4, p.1236-1242, 2001.

Van Der WERF, J.H.J.; BOER, I.J.M. Estimates of additive genetic variance when base populations are selected. Journal of Animal Science, v.68, p.3124-3132, 1990.

Recebido em: 19/09/03 Aceito em: 08/06/04 